\title{
Copper Hydride Catalyzed Synthesis of 2,3-Disubstituted Indolines
}

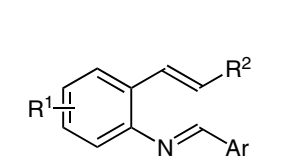

$\mathrm{Cu}(\mathrm{OAc})_{2}(4 \mathrm{~mol} \%), \mathrm{L}^{*}(4.4 \mathrm{~mol} \%)$ $\mathrm{Ph}_{3} \mathrm{P}(5 \mathrm{~mol} \%),(\mathrm{EtO}){ }_{2} \mathrm{MeSiH}$ (2 equiv) $\stackrel{t \text {-BuOD (1.1 equiv) }}{\text { MTBE-THF (95:5), r.t., } 24 \mathrm{~h}}$

Proposed catalytic cycle:

Cu(OAC)

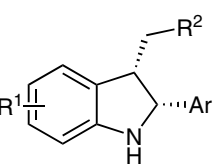

$\mathrm{dr}>99: 1$

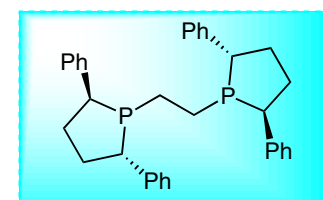

$\mathrm{L}^{*},(S, S)-\mathrm{Ph}-\mathrm{BPE}$
$L^{*},(S, S)-P h-B P E$
Category

Metal-Catalyzed

Asymmetric

Synthesis and

Stereoselective

Reactions

\section{Key words}

indolines

Markovnikov addition

organometallic reagents

copper

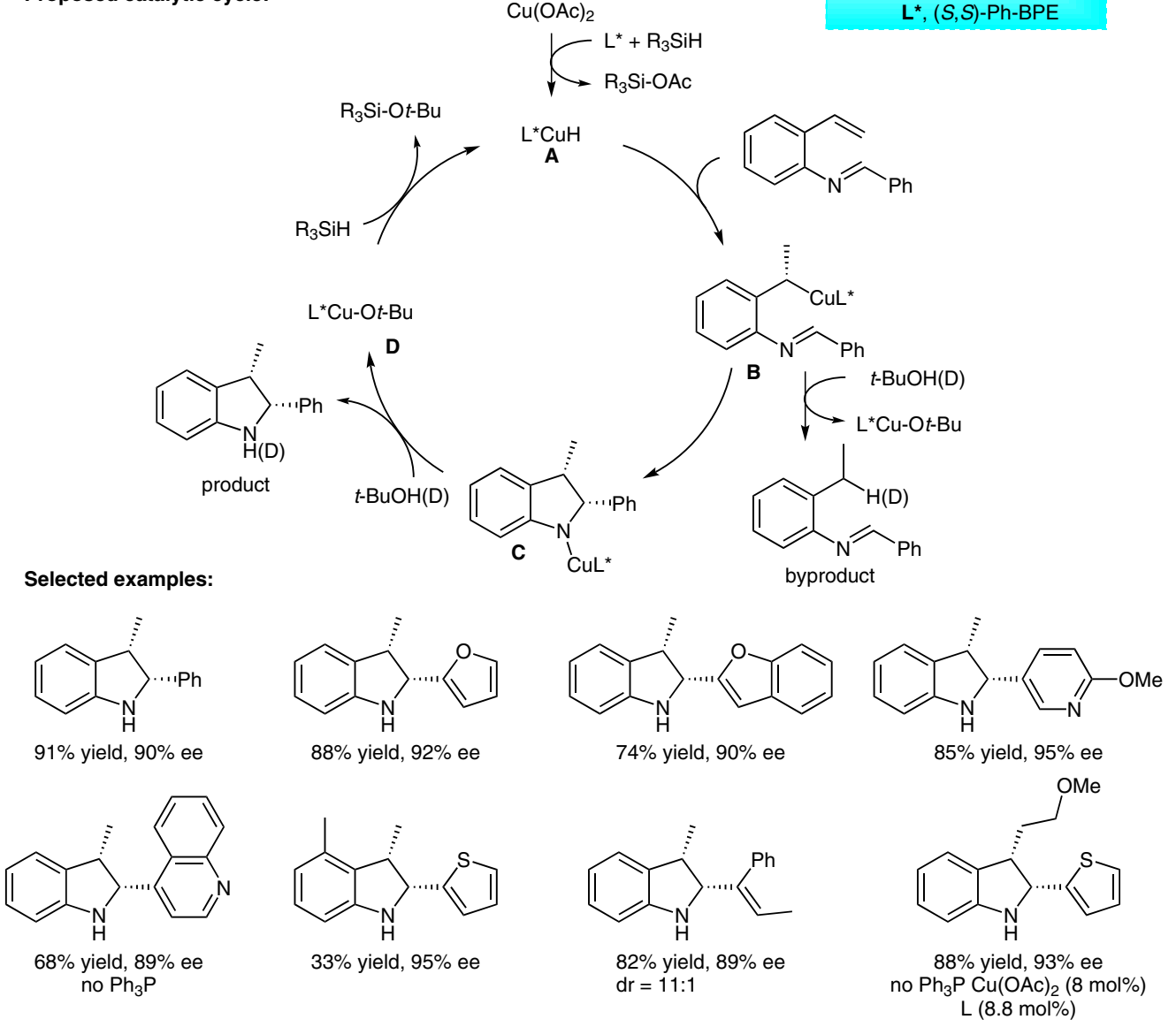

Selected examples:

$\left\lceil\mathrm{L}^{*}+\mathrm{R}_{3} \mathrm{SiH}\right.$

Significance: The $\mathrm{L}^{*} \mathrm{CuH}$-catalyzed diastereo- and enantioselective synthesis of 2,3-disubstituted cisindolines proceeds under mild conditions and tolerates bulky functional groups, including heterocycles, olefins, or substituted aromatic rings. A methyl substituent at the C-4 position of the indoline ring results in lower reactivity.
Comment: The yield of the reaction is significantly dependent on the choice of alcohol. $t$-BuOD is superior to $t-\mathrm{BuOH}$, as it reduced the amount of byproduct by slowing the protonolysis of $\mathbf{B}$. Interestingly, the reaction is highly cis-diastereoselective. Addition of triphenylphosphine as a secondary ligand significantly improves the catalyst turnover.

SYNFACTS Contributors: Hisashi Yamamoto, Biplab Maji 